\title{
Comatrix corings: Galois corings, Descent Theory, and a Structure Theorem for Cosemisimple corings
}

\author{
L. El Kaoutit and J. Gómez-Torrecillas \\ Departamento de Álgebra \\ Universidad de Granada \\ E18071 Granada, Spain \\ e-mail: kaoutit@fedro.ugr.es \\ e-mail: torrecil@ugr.es
}

\section{Introduction}

Corings and their comodules provide an appropriate formalism to unify notions and results coming from different subfields of the theory of associative algebras. One of the most striking examples is the following. Let $\psi: T \rightarrow A$ be a ring extension. M. Cipolla [8] extended Grothendieck's theory of the faithfully flat descent from the commutative case to the non commutative one. His main result says, in the restatement given in [16, Theorem 3.8], that if ${ }_{T} A$ is faithfully flat, then the tensor product functor $-\otimes_{T} A: \mathcal{M}_{T} \rightarrow$ $\operatorname{Desc}_{\psi}$ establishes an equivalence between the category $\mathcal{M}_{T}$ of all right $T$-modules and the category $D e s c_{\psi}$ of descent data. Assume now that $A$ is a right comodule algebra over a Hopf algebra $H$ and that $T$ is the subring of coinvariant elements of $A$ (see 117 ). H. J. Schneider proved that the functor $-\otimes_{A}: \mathcal{M}_{T} \rightarrow \mathcal{M}_{A}^{H}$, where $\mathcal{M}_{A}^{H}$ is the category of right Hopf $A$-modules, is an equivalence if and only if ${ }_{T} A$ is faithfully flat and the canonical map can : $A \otimes_{T} A \rightarrow A \otimes H$ is bijective [17, Theorem 1]. Both theorems have a similar flavor, and in fact they are particular cases of a recent result on corings having a grouplike element due to T. Brzeziński [4, Theorem 5.6]. The enlightening fact here is that the categories $\operatorname{Desc}_{\psi}$ and $\mathcal{M}_{A}^{H}$ are categories of comodules over suitable corings which become isomorphic to Sweedler's canonical corings of the form $A \otimes_{T} A$ (see [18]). From a categorical point of view, the $A$-corings characterized in [ [. has a structure of right comodule such that $A$ becomes a progenerator for the category of right comodules (see [ [], Theorem 2.4] and [0, Theorem 3.5], for the finitely and projective generated case). We think that the theory of corings should be developed by its own right, so, in the light of the aforementioned results, a basic question arising here is to characterize the corings whose category of comodules has a finitely generated projective generator. In order to extricate the structure of these corings, we introduce the notion of comatrix coring, which allows also to give a full generalization of Brzeziński's result which works for corings 
without grouplike elements. Comatrix corings allow as well to give a complete description of all cosemisimple corings.

We have organized our exposition as follows. After a brief introduction (Section 11) to the basic notions of corings and comodules, we expound in Section 2 how to construct a comatrix $A$-coring $\Sigma^{*} \otimes_{B} \Sigma$ from a given bimodule ${ }_{B} \Sigma_{A}$ such that $\Sigma_{A}$ is finitely generated and projective. In the case that $\Sigma$ is a right comodule over an $A$-coring $\mathfrak{C}$, we define a homomorphism of $A$-corings can : $\Sigma^{*} \otimes_{B} \Sigma \rightarrow \mathfrak{C}$. This canonical morphism generalizes the homonymous map introduced in [17].

Section 3 contains several characterizations of those corings $\mathfrak{C}$ having a finitely generated and projective generator. We prove in particular that they are comatrix corings $\Sigma^{*} \otimes_{T} \Sigma$ such that $T_{T} \Sigma$ is faithfully flat. We include some consequences, among them, we deduce «, Theorem 5.6] and a Descent Theorem for ring extensions of the form $T \rightarrow \operatorname{End}\left(\Sigma_{A}\right)$ which generalizes [8, Teorema] and [16, Theorem 3.8].

In Section 1 we offer a structure theorem for cosemisimple corings. They are described in a unique way as direct sums of comatrix corings of the form $\Sigma^{*} \otimes_{D} \Sigma$, where $D$ is a division subring of $\operatorname{End}\left(\Sigma_{A}\right)$.

Section 5 is devoted to show that comatrix corings can be alternatively introduced as coendomorphism corings.

\section{The basic notions}

We use the following conventions. For an object $C$ in a category the identity morphism $C \rightarrow C$ is denoted by $C$. We work over fixed commutative ring $K$, and all our additive categories are assumed to be $K$-linear. For instance, all rings in this paper are unitary $K$-algebras, and all bimodules are assumed to centralize the elements of $K$. Attached to every object $C$ of an additive category $\mathcal{A}$ we have its endomorphism $\operatorname{ring} \operatorname{End}_{\mathcal{A}}(C)$, whose multiplication is given the composition of the category. As usual, some special conventions will be understood for the case of endomorphism rings of modules. Thus, if $M_{R}$ is a right module over a ring $R$, then its endomorphism ring in the category $\mathcal{M}_{R}$ of all right $R$-modules will be denoted by $\operatorname{End}\left(M_{R}\right)$, while if ${ }_{R} N$ is a left $R$-module, then its endomorphism ring, denoted by $\operatorname{End}\left({ }_{R} N\right)$, is, by definition, the opposite of the endomorphism ring of $N$ in the category ${ }_{R} \mathcal{M}$ of all left modules over $R$.

Throughout this paper, $A, A^{\prime}, \ldots, B, \ldots$ denote associative and unitary algebras over a commutative ring $K$. The tensor product over $A$ is denoted by $\otimes_{A}$. We shall sometimes replace $\otimes_{K}$ by $\otimes$.

We recall from $[18 \pi$ the notion of a coring. An $A$-coring is a three-tuple $(\mathfrak{C}, \Delta, \epsilon)$ consisting of an $A$-bimodule $\mathfrak{C}$ and two $A$-bimodule maps

$$
\Delta: \mathfrak{C} \longrightarrow \mathfrak{C} \otimes_{A} \mathfrak{C}, \quad \epsilon: \mathfrak{C} \longrightarrow A
$$

such that $\left(\mathfrak{C} \otimes_{A} \Delta\right) \circ \Delta=\left(\Delta \otimes_{A} \mathfrak{C}\right) \circ \Delta$ and $\left(\epsilon \otimes_{A} \mathfrak{C}\right) \circ \Delta=\left(\mathfrak{C} \otimes_{A} \epsilon\right) \circ \Delta=\mathfrak{C}$. A right $\mathfrak{C}$-comodule is a pair $\left(M, \rho_{M}\right)$ consisting of right $A$-module $M$ and an $A$-linear map $\rho_{M}: M \rightarrow M \otimes_{A} \mathfrak{C}$ satisfying $\left(M \otimes_{A} \Delta\right) \circ \rho_{M}=\left(\rho_{M} \otimes_{A} \mathfrak{C}\right) \circ \rho_{M},\left(M \otimes_{A} \epsilon\right) \circ \rho_{M}=M ;$ such 
$M$ will be denoted by $M_{\mathfrak{C}}$. A morphism of right $\mathfrak{C}$-comodules $\left(M, \rho_{M}\right)$ and $\left(N, \rho_{N}\right)$ is a right $A$-linear map $f: M \rightarrow N$ such that $\left(f \otimes_{A} \mathfrak{C}\right) \circ \rho_{M}=\rho_{N} \circ f$; the $K$-module of all such morphisms will be denoted by $\operatorname{Hom}_{\mathfrak{C}}(M, N)$. The right $\mathfrak{C}$-comodules together with their morphisms form the additive category $\mathcal{M}^{\mathfrak{C}}$. Coproducts and cokernels in $\mathcal{M}^{\mathfrak{C}}$ do exist and can be already computed in $\mathcal{M}_{A}$. Therefore, $\mathcal{M}^{\mathfrak{C}}$ has arbitrary inductive limits. If ${ }_{A} \mathfrak{C}$ is flat, then $\mathcal{M}^{\mathfrak{C}}$ an abelian category. The converse is not true, as [10, Example 1.1] shows.

Let $\rho_{M}: M \rightarrow M \otimes_{A} \mathfrak{C}$ be a comodule structure over an $A^{\prime}-A$-bimodule $M$, and assume that $\rho_{M}$ is $A^{\prime}$-linear. For any right $A^{\prime}$-module $X$, the right $A$-linear map $X \otimes_{A^{\prime}} \rho_{M}: X \otimes_{A^{\prime}} M \rightarrow X \otimes_{A^{\prime}} M \otimes_{A} \mathfrak{C}$ makes $X \otimes_{A^{\prime}} M$ a right $\mathfrak{C}-$ comodule. This leads to an additive functor $-\otimes_{A^{\prime}} M: \mathcal{M}_{A^{\prime}} \rightarrow \mathcal{M}^{\mathfrak{c}}$. The classical adjointness isomorphism $\operatorname{Hom}_{A}\left(Y \otimes_{B} M, X\right) \cong \operatorname{Hom}_{B}\left(Y, \operatorname{Hom}_{A}(M, X)\right)$ induces, by restriction, a natural isomorphism $\operatorname{Hom}_{\mathfrak{C}}\left(Y \otimes_{B} M, X\right) \cong \operatorname{Hom}_{B}\left(Y, \operatorname{Hom}_{\mathfrak{C}}(M, X)\right)$, for $Y \in \mathcal{M}_{A^{\prime}}, X \in \mathcal{M}^{\mathfrak{c}}$. Therefore, $\operatorname{Hom}_{\mathfrak{C}}(M,-): \mathcal{M}^{\mathfrak{C}} \rightarrow \mathcal{M}_{A^{\prime}}$ is right adjoint to $-\otimes_{A^{\prime}} M: \mathcal{M}_{A^{\prime}} \rightarrow \mathcal{M}^{\mathfrak{C}}$. On the other hand, the functor $-\otimes_{A} \mathfrak{C}$ is right adjoint to the forgetful functor $U: \mathcal{M}_{A} \rightarrow \mathcal{M}^{\mathfrak{E}}$ (see [13, Proposition 3.1], [四, Lemma 3.1]).

Now assume that the $A^{\prime}-A$-bimodule $M$ is also a left $\mathfrak{C}^{\prime}$-comodule with structure map $\lambda_{M}: M \rightarrow \mathfrak{C}^{\prime} \otimes_{A} M$. It is clear that $\rho_{M}: M \rightarrow M \otimes_{A} \mathfrak{C}$ is a morphism of left $\mathfrak{C}^{\prime}$-comodules if and only if $\lambda_{M}: M \rightarrow \mathfrak{C}^{\prime} \otimes_{A^{\prime}} M$ is a morphism of right $\mathfrak{C}$-comodules. In this case, we say that $M$ is a $\mathfrak{C}^{\prime}-\mathfrak{C}$-bicomodule.

For any right $A$-module $X$, we will denote its right dual by $X^{*}=\operatorname{Hom}_{A}(X, A)$, which is a left $A$-module in a canonical way. We will use the analogous notation ${ }^{*} Y$ for left $A$ modules $Y$. There is a canonical isomorphism $\operatorname{End}\left(\mathfrak{C}_{\mathfrak{C}}\right) \cong \mathfrak{C}^{*}$ that maps an endomorphism $f$ onto $\epsilon \circ f$. The structure of ring of $\operatorname{End}\left(\mathfrak{C}_{\mathfrak{C}}\right)$ is automatically transferred to the convolution product $\mathfrak{C}^{*}$ as defined in [18, Proposition 3.2]. Analogously, there is an isomorphimsm of rings $\operatorname{End}(\mathfrak{C} \mathfrak{C})^{o p} \cong * \mathfrak{C}$. The coring $\mathfrak{C}$ becomes a ${ }^{*} \mathfrak{C}-\mathfrak{C}^{*}$-bimodule.

\section{Comatrix corings and the canonical map.}

Let $A, B$ be rings. A comatrix $A$-coring will be built on every $B-A$-bimodule which is finitely generated and projective as a right $A$-module. Sweedler's canonical corings and dual corings are examples of comatrix corings. When the bimodule enjoys a structure of right module over a given coring $\mathfrak{C}$, a canonical homomorphism of $A$-corings is shown to relate the comatrix $A$-coring and the coring $\mathfrak{C}$.

Let ${ }_{B} \Sigma_{A}$ be a $B-A$-bimodule. Then $\Sigma^{*}=\operatorname{Hom}_{A}(\Sigma, A)$ is canonically endowed with a structure of $A-B$-bimodule, and $\Sigma^{*} \otimes_{B} \Sigma$ is an $A$-bimodule in a natural way. Assume $\Sigma_{A}$ to be finitely generated and projective, and let $\left\{e_{i}^{*}, e_{i}\right\} \subseteq \Sigma^{*} \times \Sigma$ be a finite dual basis, that is, the equality

$$
u=\sum_{i} e_{i} e_{i}^{*}(u) \quad \forall u \in \Sigma
$$

holds. We can built on $\Sigma^{*} \otimes_{B} \Sigma$ a canonical structure of $A$-coring. 
2.1. Proposition. The A-bimodule $\Sigma^{*} \otimes_{B} \Sigma$ is an A-coring with comultiplication

$$
\Delta: \Sigma^{*} \otimes_{B} \Sigma \rightarrow \Sigma^{*} \otimes_{B} \Sigma \otimes_{A} \Sigma^{*} \otimes_{B} \Sigma
$$

defined by $\Delta\left(\varphi \otimes_{B} u\right)=\sum_{i} \varphi \otimes_{B} e_{i} \otimes_{A} e_{i}^{*} \otimes_{B} u$ and counit

$$
\epsilon: \Sigma^{*} \otimes_{B} \Sigma \rightarrow A
$$

given by $\epsilon\left(\varphi \otimes_{B} u\right)=\varphi(u)$. Moreover, there is a ring anti-isomorphism ${ }^{*}\left(\Sigma^{*} \otimes_{B} \Sigma\right) \cong$ $\operatorname{End}\left({ }_{B} \Sigma\right)$, where the first of these rings is the left dual of $\Sigma^{*} \otimes_{B} \Sigma$, endowed with the convolution product.

Proof. First, we should check that $\Delta$ is well-defined. This requires to prove that for every $b \in B$ and every pair $(\varphi, u) \in \Sigma^{*} \times \Sigma$ one has

$$
\sum_{i} \varphi b \otimes_{B} e_{i} \otimes_{A} e_{i}^{*} \otimes_{B} u=\sum_{i} \varphi \otimes_{B} e_{i} \otimes_{A} e_{i}^{*} \otimes_{B} b u
$$

Clearly, it suffices to show that $\sum_{i} b e_{i} \otimes_{A} e_{i}^{*}=\sum_{i} e_{i} \otimes_{A} e_{i}^{*} b$. Using (11), we compute

$$
\begin{aligned}
\sum_{i} b e_{i} \otimes_{A} e_{i}^{*}=\sum_{i, k} e_{k} e_{k}^{*}\left(b e_{i}\right) \otimes_{A} e_{i}^{*}= & \sum_{i, k} e_{k} \otimes_{A} e_{k}^{*}\left(b e_{i}\right) e_{i}^{*}= \\
& \sum_{k} e_{k} \otimes_{A}\left(\sum_{i}\left(e_{k}^{*} b\right)\left(e_{i}\right) e_{i}^{*}\right)=\sum_{k} e_{k} \otimes_{A} e_{k}^{*} b,
\end{aligned}
$$

as desired. It is now routine to check that $\Delta$ and $\epsilon$ are homomorphisms of $A$-bimodules. An easy computation gives the coassociative and counitary properties. The reader should realize that (1) is used again in the proof of the counitary property. Finally, let us prove the stated ring isomorphism. The isomorphism is given, at the level of $K$-modules, by the composition

$$
{ }^{*}\left(\Sigma^{*} \otimes_{B} \Sigma\right)=\operatorname{Hom}_{A}\left(\Sigma^{*} \otimes_{B} \Sigma,{ }_{A} A\right) \cong \operatorname{Hom}_{B}\left(\Sigma,{ }^{*}\left(\Sigma^{*}\right)\right) \cong \operatorname{Hom}_{B}(\Sigma, \Sigma),
$$

where we have used one adjointness isomorphism and the canonical isomorphism $\Sigma \cong$ ${ }^{*}\left(\Sigma^{*}\right)$. By computing explicitly this composition is given by the assignment $f \mapsto \hat{f}$, where $\hat{f}: \Sigma \rightarrow \Sigma$ is given by

$$
\hat{f}(u)=\sum_{i} e_{i} f\left(e_{i}^{*} \otimes_{B} u\right) \text {, for every } f \in{ }^{*}\left(\Sigma^{*} \otimes_{B} \Sigma\right) .
$$

We are now ready to check that $\widehat{(-)}$ is a ring anti-homomorphism. First, we have $\hat{\epsilon}(u)=$ $\sum_{i} e_{i} \epsilon\left(e_{i}^{*} \otimes_{B} u\right)=\sum_{i} e_{i} e_{i}^{*}(u)=u$, for every $u \in \Sigma$. Given, $f, g \in{ }^{*}\left(\Sigma^{*} \otimes_{B} \Sigma\right)$, the convolution product reads

$$
(f * g)\left(\varphi \otimes_{B} u\right)=f\left(I \otimes_{A} g\right) \Delta\left(\varphi \otimes_{B} u\right)=\sum_{i} f\left(\varphi \otimes_{B} e_{i} g\left(e_{i}^{*} \otimes_{B} u\right)\right) .
$$


Therefore,

$$
\begin{aligned}
\widehat{f * g}(u)=\sum_{j} e_{j}(f * g)\left(e_{j}^{*} \otimes_{B} u\right)=\sum_{i, j} e_{j} f\left(e_{j}^{*} \otimes_{B} e_{i} g\left(e_{i}^{*} \otimes_{B} u\right)\right)= \\
\hat{f}\left(\sum_{i} e_{i} g\left(e_{i}^{*} \otimes_{B} u\right)\right)=\hat{f}(\hat{g}(u)),
\end{aligned}
$$

and we get that $\widehat{f * g}=\hat{f} \circ \hat{g}$. Since the product in $\operatorname{End}\left({ }_{B} \Sigma\right)$ is the opposite of the composition, we have already proved that our map is an anti-isomorphism of rings.

2.2. Remark. Let $\left\{e_{i}^{*}, e_{i}\right\}_{1 \leq i \leq n}$ and $\left\{f_{j}^{*}, f_{j}\right\}_{1 \leq j \leq m}$ be two dual bases for $\Sigma_{A}$. The wellknown isomorphism of $\operatorname{End}\left(\Sigma_{A}\right)$-bimodules

$$
\begin{aligned}
& \Sigma \otimes_{A} \Sigma^{*} \stackrel{\xi}{\longrightarrow} \operatorname{End}\left(\Sigma_{A}\right) \\
& u \otimes_{A} \varphi \longmapsto[v \mapsto u \varphi(v)]
\end{aligned}
$$

easily shows that $\sum_{1 \leq i \leq n} e_{i} \otimes_{A} e_{i}^{*}=\sum_{1 \leq j \leq m} f_{j} \otimes_{A} f_{j}^{*}$ and, hence, the comultiplication of the comatrix $A$-coring $\Sigma^{*} \otimes_{B} \Sigma$ does not depend on the choice of the dual basis of $\Sigma_{A}$.

Our comatrix corings generalize two fundamental classes of corings.

2.3. Example (Sweedler's canonical coring). Let $B \rightarrow A$ a ring homomorphism. The bimodule ${ }_{B} A_{A}$ is projective and finitely generated as a right $A$-module. The corresponding comatrix $A$-coring is then isomorphic to the canonical Sweedler's coring $A \otimes_{B} A[18$, Example 1.2]. The comultiplication sends $a \otimes_{B} a^{\prime}$ onto $a \otimes_{B} 1 \otimes_{A} 1 \otimes_{B} a^{\prime}$, and the counit is given by the multiplication of $A$.

2.4. Example (Dual coring). Let $A \rightarrow B$ a ring homomorphism. Assume that $B_{A}$ is finitely generated and projective. Then, taking the dual with respect to $A$, we have $B^{*} \otimes_{B} B \cong B^{*}$, and this isomorphism of $A$-bimodules becomes an isomorphism of $A$ corings whenever we consider the $A$-coring structure on $B^{*}$ obtained from the $A$-ring structure of $B$ [18, 3.7].

A relevant feature of the $A$-coring $\Sigma^{*} \otimes_{B} \Sigma$ is that the right $A$-module $\Sigma$ becomes a right $\Sigma^{*} \otimes_{B} \Sigma$-comodule in a canonical way. Its coaction is defined as

$$
\Sigma \stackrel{\rho_{\Sigma}}{\longrightarrow} \Sigma \otimes_{A} \Sigma^{*} \otimes_{B} \Sigma, \quad\left(u \longmapsto \sum_{i} e_{i} \otimes_{A} e_{i}^{*} \otimes_{B} u\right)
$$

which is clearly a left $B$-linear map, in other words, $\Sigma$ is $B-\left(\Sigma^{*} \otimes_{B} \Sigma\right)$-bicomodule. This comodule plays a relevant role. 
2.5. Proposition. The coring $\Sigma^{*} \otimes_{B} \Sigma$ is, as a right comodule, generated by $\Sigma$. Therefore, every right $\Sigma^{*} \otimes_{B} \Sigma$-comodule is isomorphic to a subcomodule of a quotient of $\Sigma^{(I)}$, for a suitable index set I. Moreover,

$$
\operatorname{End}\left(\Sigma_{\Sigma^{*} \otimes_{B} \Sigma}\right)=\left\{f \in \operatorname{End}\left(\Sigma_{A}\right) \mid f \otimes_{B} x=1 \otimes_{B} f(x), \text { for every } x \in \Sigma\right\}
$$

and, in particular, the canonical ring homomorphism $B \rightarrow \operatorname{End}\left(\Sigma_{A}\right)$ factorizes throughout $\operatorname{End}\left(\Sigma_{\Sigma^{*} \otimes_{B} \Sigma}\right)$.

Proof. For the first statement, it is enough to prove that every generator $\varphi \otimes_{B} u \in \Sigma^{*} \otimes_{B} \Sigma$ belongs to the image of a morphism of right $\Sigma^{*} \otimes_{B} \Sigma$-comodules $f: \Sigma \rightarrow \Sigma^{*} \otimes_{B} \Sigma$. This is fulfilled by the map defined by $f(u)=\varphi \otimes_{B} u$, which is easily proved to be a homomorphism of comodules. For the second statement, let $\rho_{M}: M \rightarrow M \otimes_{A} \Sigma^{*} \otimes_{B} \Sigma$ be a right comodule. The own structure map $\rho_{M}$ is a morphism of comodules which splits as a right $A$-module map. Therefore, $M$ is isomorphic to a subcomodule of $M \otimes_{A} \Sigma^{*} \otimes_{B} \Sigma$, which is now easily shown to be a quotient of a coproduct of copies of $\Sigma$. The second statement follows from a straightforward computation.

2.6. Remark. Of course $\Sigma^{*}$ is a left $\Sigma^{*} \otimes_{B} \Sigma$-comodule with a right $B$-linear coaction

$$
\Sigma^{*} \stackrel{\lambda_{\Sigma^{*}}}{\longrightarrow} \Sigma^{*} \otimes_{B} \Sigma \otimes_{A} \Sigma^{*}, \quad\left(\varphi \mapsto \sum_{i} \varphi \otimes_{B} e_{i} \otimes_{A} e_{i}^{*}\right) .
$$

Moreover, $\Sigma^{*} \otimes_{B} \Sigma^{\Sigma^{*}}$ satisfies the left version of Proposition 2.5, and the right convolution ring $\left(\Sigma^{*} \otimes_{B} \Sigma\right)^{*}$ is a ring anti-isomorphic to $\operatorname{End}\left(\Sigma^{*} B\right)$.

Now, let $\mathfrak{C}$ be any $A$-coring, and assume $\Sigma$ to be a right $\mathfrak{C}$-comodule with coaction $\rho_{\Sigma}: \Sigma \rightarrow \Sigma \otimes_{A} \mathfrak{C}$. From now on, we will denote $S=\operatorname{End}\left(\Sigma_{A}\right)$ and $T=\operatorname{End}\left(\Sigma_{\mathfrak{C}}\right)$. Then $\Sigma$ becomes an $S-A$-bimodule and $\rho_{\Sigma}$ is a homomorphism of $S-A$-bimodules. We keep in mind that $T$ is a subring of $S$.

2.7. Proposition. If $\Sigma_{\mathfrak{C}}$ is a comodule such that $\Sigma_{A}$ is finitely generated and projective, then the map can : $\Sigma^{*} \otimes_{T} \Sigma \rightarrow \mathfrak{C}$ defined as the composition

$$
\Sigma^{*} \otimes_{T} \Sigma \stackrel{\Sigma^{*} \otimes_{B} \rho_{\Sigma}}{\longrightarrow} \Sigma^{*} \otimes_{T} \Sigma \otimes_{A} \mathfrak{C} \stackrel{\epsilon \otimes_{A} \mathfrak{C}}{\longrightarrow} A \otimes_{A} \mathfrak{C} \cong \mathfrak{C}
$$

is a homomorphism of A-corings.

Proof. By construction can is $A$-bilinear. We need to check the identities

$$
\Delta_{\mathfrak{C}} \circ \operatorname{can}=\left(\operatorname{can} \otimes_{A} c a n\right) \circ \Delta \quad \text { and } \quad \epsilon_{\mathfrak{C}} \circ \text { can }=\epsilon .
$$

If $\left\{e_{i}^{*}, e_{i}\right\} \subseteq \Sigma^{*} \times \Sigma$ is a dual basis, then $\rho_{\Sigma}\left(e_{j}\right)=\sum_{i} e_{i} \otimes_{A} \rho_{i j}$ for each $j$. Then the first identity in (5) is equivalent to

$$
\sum_{k} e_{i}^{*}\left(e_{k}\right) \Delta_{\mathfrak{C}}\left(\rho_{k j}\right)=\sum_{k, l, m} e_{i}^{*}\left(e_{l}\right) \rho_{l k} \otimes_{A} e_{k}^{*}\left(e_{m}\right) \rho_{m j} \quad \forall i, j
$$


The fact that $\Sigma$ is a right $\mathfrak{C}$-comodule gives

$$
\sum_{k} e_{k} \otimes_{A} \Delta_{\mathfrak{C}}\left(\rho_{k j}\right)=\sum_{k, l} e_{k} \otimes_{A} \rho_{k l} \otimes_{A} \rho_{l j} \quad \forall j
$$

Therefore,

$$
\sum_{k} e_{i}^{*}\left(e_{k}\right) \Delta_{\mathfrak{C}}\left(\rho_{k j}\right)=\sum_{k, l} e_{i}^{*}\left(e_{k}\right) \rho_{k l} \otimes_{A} \rho_{l j} \quad \forall i, j
$$

On the other hand,

$$
\rho_{\Sigma}\left(e_{m}\right)=\rho_{\Sigma}\left(\sum_{k} e_{k} e_{k}^{*}\left(e_{m}\right)\right)=\sum_{k} \rho_{\Sigma}\left(e_{k}\right) e_{k}^{*}\left(e_{m}\right)=\sum_{l, k} e_{l} \otimes_{A} \rho_{l k} e_{k}^{*}\left(e_{m}\right) \quad \forall m
$$

thus

$$
\sum_{l} e_{l} \otimes_{A} \rho_{l m}=\sum_{l, k} e_{l} \otimes_{A} \rho_{l k} e_{k}^{*}\left(e_{m}\right) \quad \text { and } \quad \sum_{l} e_{i}^{*}\left(e_{l}\right) \rho_{l m}=\sum_{l, k} e_{i}^{*}\left(e_{l}\right) \rho_{l k} e_{k}^{*}\left(e_{m}\right) \quad \forall i, m
$$

Finally, we get

$$
\sum_{k, l, m} e_{i}^{*}\left(e_{l}\right) \rho_{l k} \otimes_{A} e_{k}^{*}\left(e_{m}\right) \rho_{m j}=\sum_{k, l, m} e_{i}^{*}\left(e_{l}\right) \rho_{l k} e_{k}^{*}\left(e_{m}\right) \otimes_{A} \rho_{m j}=\sum_{l, m} e_{i}^{*}\left(e_{l}\right) \rho_{l m} \otimes_{A} \rho_{m j} \quad \forall i, j
$$

This implies, in view of (7), the identity (6).

The second identity in (5) holds since, for every $i, j$, we have

$$
\begin{aligned}
\epsilon_{\mathfrak{C}}\left(\operatorname{can}\left(e_{i}^{*} \otimes e_{j}\right)\right)=\epsilon_{\mathfrak{C}}\left(\sum_{k} e_{i}^{*}\left(e_{k}\right) \rho_{k j}\right)=\sum_{k} e_{i}^{*}\left(e_{k}\right) \epsilon_{\mathfrak{C}}\left(\rho_{k j}\right) & \\
& =e_{i}^{*}\left(\sum_{k} e_{k} \epsilon_{\mathfrak{C}}\left(\rho_{k j}\right)\right)=e_{i}^{*}\left(e_{j}\right)=\epsilon\left(e_{i}^{*} \otimes e_{j}\right)
\end{aligned}
$$

The following examples suggest that the can map defined in Proposition 2.7 is an interesting object for research. Moreover, it generalizes canonical maps previously considered in the theories of Hopf modules and noncommutative Galois extensions.

2.8. Example. Let us assume that our $A$-coring $\mathfrak{C}$ has a grouplike element $g$, which is equivalent, by $\llbracket$, Lemma 5.1], to endow $A$ with a structure of right comodule over $\mathfrak{C}$. In this case $T=\operatorname{End}\left(A_{\mathfrak{C}}\right)$ is nothing but the subring of coinvariants [5, Proposition 2.2] of $A$. In this case, the map can $: A \otimes_{T} A \rightarrow \mathfrak{C}$ is determined by the condition $\operatorname{can}\left(1 \otimes_{T} 1\right)=g$. Therefore, the coring $\mathfrak{C}$ is Galois in the sense of $₫$, Definition 5.3] if and only if can is an isomorphism. It is convenient to point out here that our homomorphism can generalizes the original canonical map considered by [17] and, in fact, the map can defined in [3, Definition 2.1]. 
2.9. Example. Let $G$ be a finite group of ring automorphims of $A$, and let $R=G * A$ be associated crossed product. The ring $A$ embeds canonically in $R$ and, by construction, $R_{A}$ is free with basis $G$ and we can consider the corresponding comatrix coring $R^{*} \otimes_{R} R \cong R^{*}$ (see Example 2.4). Let us show that the "trace map" $g: R \rightarrow A$ defined by $g\left(\sum_{\sigma \in G} \sigma a_{\sigma}\right)=$ $\sum_{\sigma \in G} a_{\sigma}$ is a grouplike element for $R^{*}$. Accordingly with [15, Theorem 3], we need just to check that $g$ acts as the identity on $A$, which is obviously the case, and that $K \operatorname{erg}$ is a right ideal of $R$. This last condition can be checked in a straightforward way taking that the trace map is invariant under translations into account. Thus, $A$ is a right $R^{*}$ comodule and we have the homomorphism of $A$-corings can : $A \otimes_{T} A \rightarrow R^{*}$ determined by $\operatorname{can}\left(1 \otimes_{T} 1\right)=g$, where $T$ is the subring of $g$-coinvariants of $A$. An easy computation shows that $T$ is already the subring of $G$-invariants of $A$. Now, the composite homomorphism of rings

$$
R \cong{ }^{*}\left(R^{*}\right) \stackrel{{ }^{*} \operatorname{can}}{\longrightarrow}{ }^{*}\left(A \otimes_{T} A\right) \cong \operatorname{End}\left({ }_{T} A\right)
$$

is precisely the map $\delta$ defined in [14] (or $j$ in [9]). There, the extension $T \subseteq A$ is said to be $G$-Galois whenever $\delta$ is an isomorphism and ${ }_{T} A$ is finitely generated and projective. Of course, $\delta$ is an isomorphism if and only if can is an isomorphism.

The homomomorphism of $A$-corings can $: \Sigma^{*} \otimes_{T} \Sigma \rightarrow \mathfrak{C}$ leads to the functor

$$
C A N: \mathcal{M}^{\Sigma^{*} \otimes_{T} \Sigma} \rightarrow \mathcal{M}^{\mathfrak{C}}
$$

which sends a comodule $\rho_{M}: M \rightarrow M \otimes_{A} \Sigma^{*} \otimes_{T} \Sigma$ onto the $\mathfrak{C}$-comodule

$$
M \stackrel{\rho_{M}}{\longrightarrow} M \otimes_{A} \Sigma^{*} \otimes_{T} \Sigma \stackrel{M \otimes_{A} \text { can }}{\longrightarrow} M \otimes_{A} \mathfrak{C}
$$

This is an example of induction functor (see [11, 5.2] for details).

2.10. Proposition. If $\Sigma_{\mathfrak{C}}$ is a comodule such that $\Sigma_{A}$ is finitely generated and projective, then $T=\operatorname{End}\left(\Sigma_{\Sigma^{*} \otimes_{T} \Sigma}\right)$ and we have a commuting diagram of functors

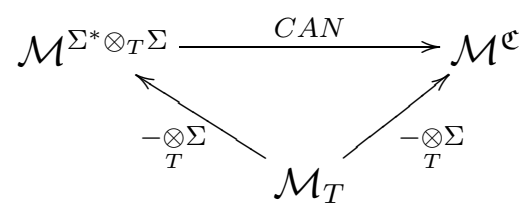

Proof. By Proposition 2.5, $T \subseteq \operatorname{End}\left(\Sigma_{\Sigma^{*} \otimes_{T} \Sigma}\right)$. Conversely, let $f \in \operatorname{End}\left(\Sigma_{\Sigma^{*} \otimes_{T} \Sigma}\right)$; the following diagram is clearly commutative

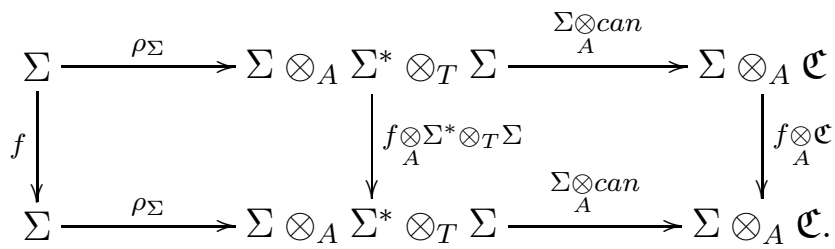


Now, an easy computation shows that $\left(\Sigma \otimes_{A}\right.$ can $) \rho_{\Sigma}$ is just the structure map for $\Sigma_{\mathfrak{C}}$. Thus $f$ is right $\mathfrak{C}$-colinear, that is, $f \in T$. Observe that we have already shown that $C A N\left(\Sigma_{\Sigma^{*} \otimes_{T} \Sigma}\right)=\Sigma_{\mathfrak{C}}$. This implies that $C A N\left(\left(X \otimes_{T} \Sigma\right)_{\Sigma^{*} \otimes_{T} \Sigma}\right)=\left(X \otimes_{T} \Sigma\right)_{\mathfrak{C}}$ for every $X \in \mathcal{M}_{T}$, as desired.

\section{Corings with a finitely generated and projective generator}

We give a complete description in terms of comatrix corings of corings having a finitely generated projective generator. Furthermore, our result generalizes [■, Theorem 5.6] to corings which possibly have not grouplike elements and, therefore, it is ultimately a generalization of [17, Theorem 1] and [8, Teorema].

Let $\Sigma$ be a right comodule over an $A$-coring $\mathfrak{C}$, and let $T=\operatorname{End}\left(\Sigma_{\mathfrak{C}}\right)$ its endomorphism ring. The structure map of $\Sigma$ is $T$-linear and, thus, we have the functor $-\otimes_{T} \Sigma: \mathcal{M}_{T} \rightarrow \mathcal{M}^{\mathfrak{C}}$. Recall that $\operatorname{Hom}_{\mathfrak{C}}(\Sigma,-): \mathcal{M}^{\mathfrak{C}} \rightarrow \mathcal{M}_{T}$ is right adjoint to $-\otimes_{T} \Sigma$. Let $\chi: \operatorname{Hom}_{\mathfrak{C}}(\Sigma,-) \otimes_{T} \Sigma \rightarrow 1$ the counit of this adjunction.

The proof of our main theorem will be better understood if we isolate a technical fact which, in view of [0, Proposition 1.1] and [1, Theorem 2.2] seems to be of independent interest.

3.1. Lemma. Let $\Sigma$ be a right $\mathfrak{C}$-comodule such that $\Sigma_{A}$ is finitely generated and projective. The counit $\chi_{\mathfrak{C}}$ at $\mathfrak{C}$ is an isomorphism if and only if can is an isomorphism.

Proof. Making use of the isomorphism $\operatorname{Hom}_{\mathfrak{C}}(\Sigma, \mathfrak{C}) \cong \Sigma^{*}$, we have that can $: \Sigma^{*} \otimes_{T} \Sigma \rightarrow \mathfrak{C}$ can be written as the composite

$$
\Sigma^{*} \otimes_{T} \Sigma \cong \operatorname{Hom}_{\mathfrak{C}}(\Sigma, \mathfrak{C}) \otimes_{T} \Sigma \stackrel{\chi_{\mathfrak{C}}}{\longrightarrow} \mathfrak{C}
$$

3.2. Theorem. Let $\mathfrak{C}$ be an $A$-coring, and $\Sigma_{\mathfrak{C}}$ a right $\mathfrak{C}$-comodule. Consider the ring extension $T \subseteq S$, where $T=\operatorname{End}\left(\Sigma_{\mathfrak{C}}\right)$ and $S=\operatorname{End}\left(\Sigma_{A}\right)$. The following statements are equivalent

(i) ${ }_{A} \mathfrak{C}$ is flat and $\Sigma_{\mathfrak{C}}$ is a finitely generated and projective generator for $\mathcal{M}^{\mathfrak{C}}$;

(ii) ${ }_{A} \mathfrak{C}$ is flat, $\Sigma_{A}$ is a finitely generated and projective $A$-module, can : $\Sigma^{*} \otimes_{T} \Sigma \rightarrow \mathfrak{C}$ is an isomorphism of $A$-corings, and $-\otimes_{T} \Sigma: \mathcal{M}_{T} \rightarrow \mathcal{M}^{\Sigma^{*} \otimes_{T} \Sigma}$ is an equivalence of categories;

(iii) $\Sigma_{A}$ is a finitely generated and projective $A$-module, can : $\Sigma^{*} \otimes_{T} \Sigma \rightarrow \mathfrak{C}$ is an isomorphism of $A$-corings, and ${ }_{T} \Sigma$ is a faithfully flat module. 
(iv) ${ }_{A} \mathfrak{C}$ is flat, $\Sigma_{A}$ is a finitely generated and projective $A-$ module, can : $\Sigma^{*} \otimes_{T} \Sigma \rightarrow \mathfrak{C}$ is an isomorphism of $A$-corings, and ${ }_{T} S$ is faithfully flat.

Proof. $(i) \Rightarrow($ ii $)$ Since ${ }_{A} \mathfrak{C}$ is flat, it follows from [10, Proposition 1.2] that $\mathcal{M}^{\mathfrak{C}}$ is a Grothendieck category and the forgetful functor $U: \mathcal{M}^{\mathfrak{C}} \rightarrow \mathcal{M}_{A}$ is exact. Moreover, it has an exact right adjoint $-\otimes_{A} \mathfrak{C}$. This implies that $\Sigma_{A}$ is finitely generated and projective. Recall that $\operatorname{Hom}_{\mathfrak{C}}(\Sigma,-): \mathcal{M}^{\mathfrak{C}} \rightarrow \mathcal{M}_{T}$ is right adjoint to $-\otimes_{T} \Sigma$ and, since $\Sigma_{\mathfrak{C}}$ is a finitely generated and projective generator, it is already an equivalence of categories. In particular, the counity of the adjunction $\chi: \operatorname{Hom}_{\mathfrak{C}}(\Sigma,-) \otimes_{T} \Sigma \rightarrow 1$ is a natural isomorphism. By Lemma 3.1, $C A N: \mathcal{M}^{\Sigma^{*} \otimes_{T} \Sigma} \rightarrow \mathcal{M}^{\mathfrak{C}}$ is an equivalence of categories. By Proposition 2.10, we have that $-\otimes_{T} \Sigma: \mathcal{M}_{T} \rightarrow \mathcal{M}^{\Sigma^{*} \otimes_{T} \Sigma}$ is an equivalence of categories.

(ii) $\Rightarrow$ (iii) The functor $-\otimes_{T} \Sigma: \mathcal{M}_{T} \rightarrow \mathcal{M}^{\Sigma^{*} \otimes_{T} \Sigma}$ is obviously faithful and exact. Since $\Sigma^{*} \otimes_{T} \Sigma \cong \mathfrak{C}$ is flat as a left $A$-module, we have, by [10, Proposition 1.2 ], that the forgetful functor $U: \mathcal{M}^{\Sigma^{*} \otimes_{T} \Sigma} \rightarrow \mathcal{M}_{A}$ is faithful and exact. Therefore, the functor $-\otimes_{T} \Sigma: \mathcal{M}_{T} \rightarrow$ $\mathcal{M}_{A}$ is faithful and exact, that is, ${ }_{T} \Sigma$ is a faithfully flat module.

$($ iii $) \Rightarrow(i)$. Let $\Omega=\Sigma^{*} \otimes_{T} \Sigma$, which is flat as a left $A$-module because ${ }_{T} \Sigma$ is flat. The

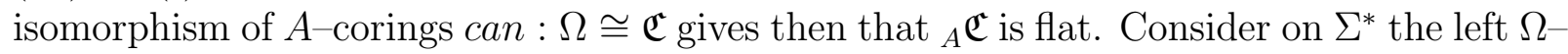
comodule structure given in Remark 2.6. A straightforward computation shows that $\Sigma^{*}$ is an $\Omega-T$-bicomodule, where $T$ acts canonically on $\Sigma^{*}$. The functor $-\otimes_{A} \Sigma^{*}: \mathcal{M}_{A} \rightarrow \mathcal{M}_{T}$ is right adjoint to $-\otimes_{T} \Sigma: \mathcal{M}_{T} \rightarrow \mathcal{M}_{A}$. By [11, Proposition 4.2], the cotensor product functor $-\square_{\Omega} \Sigma^{*}: \mathcal{M}^{\Omega} \rightarrow \mathcal{M}_{T}$ is right adjoint to $-\otimes_{T} \Sigma: \mathcal{M}_{T} \rightarrow \mathcal{M}^{\Omega}$. Since ${ }_{T} \Sigma$ is flat we have, by [11, Lemma 2.2], the isomorphism $\left(M \square_{\Omega} \Sigma^{*}\right) \otimes_{T} \Sigma \cong M \square_{\Omega}\left(\Sigma^{*} \otimes_{T} \Sigma\right)=M \square_{\Omega} \Omega \cong M$, which turns out to be inverse to the counity of the adjunction at $M \in \mathcal{M}^{\Omega}$. Moreover, if $\eta_{X}$ : $X \rightarrow\left(X \otimes_{T} \Sigma\right) \square_{\Omega} \Sigma^{*}$ is the unity of the adjunction at $X \in \mathcal{M}_{T}$, then an inverse to $\eta_{X} \otimes_{T} \Sigma$ is obtained by the isomorphism $\left.\left(\left(X \otimes_{T} \Sigma\right) \square_{\Omega} \Sigma^{*}\right)\right) \otimes_{T} \Sigma \cong\left(X \otimes_{T} \Sigma\right) \square_{\Omega}\left(\Sigma^{*} \otimes_{T} \Sigma\right) \cong X \otimes_{T} \Sigma$. Since ${ }_{T} \Sigma$ is faithful, we get that $\eta_{X}$ is an isomorphism and, hence, $-\otimes_{T} \Sigma: \mathcal{M}_{T} \rightarrow \mathcal{M}^{\Omega}$ is an equivalence of categories with inverse $-\square_{\Omega} \Sigma^{*}$. It follows from Proposition 2.10 that $-\otimes_{T} \Sigma: \mathcal{M}_{T} \rightarrow \mathcal{M}^{\mathfrak{C}}$ is an equivalence, as can $: \Omega \rightarrow \mathfrak{C}$ is an isomorphism. Therefore, $\Sigma_{\mathfrak{C}}$ is a finitely generated and projective generator for $\mathcal{M}^{\mathfrak{C}}$.

$($ iii $) \Rightarrow($ iv $)$. The exact and faithful functor $-\otimes_{T} \Sigma: \mathcal{M}_{T} \rightarrow \mathcal{M}_{A}$ decomposes as $-\otimes_{T} \Sigma \simeq$ $\left(-\otimes_{S} \Sigma\right) \circ\left(-\otimes_{T} S\right)$. This implies that $-\otimes_{T} S$ is an exact and faithful functor, as $-\otimes_{S} \Sigma$ is faithful (because $\Sigma_{A}$ is finitely generated and projective). Therefore, ${ }_{T} S$ is faithfully flat. $(i v) \Rightarrow(i i i)$. It suffices to show that ${ }_{T} \Sigma$ is faithfully flat. Consider a short exact sequence in $\mathcal{M}_{T}, 0 \longrightarrow Y \stackrel{f}{\longrightarrow} Y^{\prime} \stackrel{g}{\longrightarrow} Y^{\prime \prime} \longrightarrow 0$, and let $Z$ denote the kernel of the morphism $f \otimes_{T} \Sigma$ in the category $\mathcal{M}^{\Sigma^{*} \otimes_{T} \Sigma}$. Since the forgetful functor $\mathcal{M}^{\Sigma^{*} \otimes_{T} \Sigma} \rightarrow \mathcal{M}_{A}$ is exact, this kernel coincides with the kernel computed in $\mathcal{M}_{A}$. We thus get a commutative diagram with exact rows

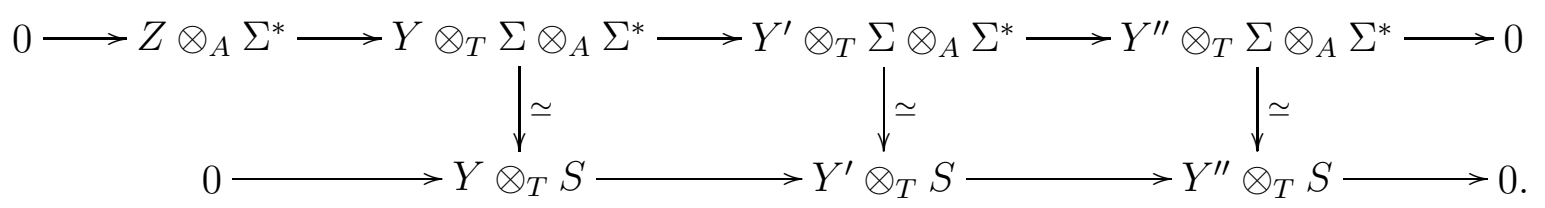

Therefore, $Z \otimes_{A} \Sigma^{*}=0$, which implies that $Z \otimes_{A} \Sigma^{*} \otimes_{T} \Sigma=0$, and thus $Z=0$, since $Z \in$ 
$\mathcal{M}^{\Sigma^{*} \otimes_{T} \Sigma}$. Therefore, ${ }_{T} \Sigma$ is flat. Now, given any right $T$-module $Y$ such that $Y \otimes_{T} \Sigma=0$, we have $Y \otimes_{T} \Sigma \otimes_{A} \Sigma^{*} \cong Y \otimes_{T} S=0$, and so $Y=0$. Thus, ${ }_{T} \Sigma$ is a faithfully flat module, and this finishes the proof.

3.3. Remark. The flatness of ${ }_{A} \mathfrak{C}$ cannot be dropped in the statements (ii) and (iv). Counterexamples can be obtained as follows. Let $e \in A$ an idempotent and write $f=1-e$. Assume that $f A e=0$ and let $I=e A$. Then $I$ becomes an $A$-coring whose comultiplication is given by the canonical isomorphism $I \cong I \otimes_{A} I$ and the counit is just the inclusion $I \subseteq A$. In this case, $\operatorname{End}\left(I_{I}\right)=\operatorname{End}\left(I_{A}\right) \cong e A e$, and, hence, $T=S$. Moreover, this easily implies that can : $I^{*} \otimes_{e A e} I \cong A e \otimes_{e A e} I \cong I$. The right $I$-comodules are those right $A$-modules satisfying that the canonical map $M \otimes_{A} I \rightarrow M$ is an isomorphism. This allows to prove that $-\otimes_{e A e} I: \mathcal{M}_{e A e} \rightarrow \mathcal{M}^{I}$ is an equivalence of categories. However, ${ }_{A} I$ is not flat unless $e A e I$ is.

Next, we shall locate Theorem 3.2 within the recent developments Galois corings with grouplike elements.

3.4. Definition. Let $\mathfrak{C}$ be an $A$-coring with a right $\mathfrak{C}$-comodule $\Sigma$ such that $\Sigma_{A}$ is finitely generated and projective. The coring will be said to be Galois if can $: \Sigma^{*} \otimes_{T} \Sigma \rightarrow \mathfrak{C}$ is an isomorphism, where $T=\operatorname{End}\left(\Sigma_{\mathfrak{C}}\right)$. When $\Sigma=A$, this definition coincides with the given by T. Brzeziński for corings with a grouplike element [四, Definition 5.3]. In view of Theorem 3.2, a ring extension of the form $T \rightarrow \operatorname{End}\left(\Sigma_{A}\right)$ could be called a $\mathfrak{C}$-Galois ring extension whenever $\Sigma$ is a right $\mathfrak{C}$-comodule such that $\Sigma_{A}$ is finitely generated and projective and $T=\operatorname{End}\left(\Sigma_{\mathfrak{C}}\right)$. Coring Galois extensions in [4] or [7] are then obtained with $\Sigma=A$.

We easily get now.

3.5. Corollary. [A, Theorem 5.6] Let $\mathfrak{C}$ be an A-coring with a grouplike element, and $T$ be the subring of coinvariant elements of $A$. If $\mathfrak{C}$ is Galois and ${ }_{T} A$ is faithfully flat, then $-\otimes_{T} A: \mathcal{M}_{T} \rightarrow \mathcal{M}^{\mathfrak{C}}$ is an equivalence of categories. Conversely, if $-\otimes_{T} A$ is an equivalence of categories, then $\mathfrak{C}$ is Galois. In this case if ${ }_{A} \mathfrak{C}$ is flat, then ${ }_{T} A$ is faithfully flat.

Proof. Put $\Sigma=A$. The corollary follows from Example 2.8, Proposition 2.10, Lemma 3.1 and, mainly, Theorem 3.2.

Galois corings with grouplike element has been also recently considered from the point of view of category equivalences by J. Y. Abuhlail [1]. Some of his results can be easily derived from our set up.

3.6. Remark. In view of Lemma 3.1, can is an isomorphism if and only if $\chi_{\mathfrak{C}}$ is an isomorphism. Taking $\Sigma=A$ in Theorem 3.2 we obtain that for if $\mathfrak{C}$ has a grouplike element and ${ }_{A} \mathfrak{C}$ is flat then $A$ is a projective generator for $\mathcal{M}^{\mathfrak{C}}$ if and only if ${ }_{T} A$ is faithfully flat and $\chi_{\mathfrak{C}}$ is bijective. This has been recently proved by J. Y. Abuhlail under the additional condition " ${ }_{A} \mathfrak{C}$ is locally projective" (see [1, Theorem 2.4]). 
3.7. Remark. It follows from Lemma 3.1 and from the proof of $(i i i) \Rightarrow(i)$ in Theorem 3.2 that the counit $\chi_{M}$ is an isomorphism for every $M \in \mathcal{M}^{\mathfrak{E}}$ if and only if ${ }_{T} \Sigma$ is flat and $\mathfrak{C}$ is Galois. Taking $\Sigma=A$, we get that the coring $\mathfrak{C}$ with a grouplike satisfies the "Weak Structure Theorem" if and only if ${ }_{T} A$ is flat and $\mathfrak{C}$ is Galois. This has been proved in [1, Theorem 2.2] under the additional condition " ${ }_{A} \mathfrak{C}$ is locally projective".

The interest in corings has been partly recovered because the theory of entwined modules (and, henceforth, of Hopf modules) can be subsumed in the theory of comodules over certain corings 四, Proposition 2.2].

3.8. Example. Let $(A, C)_{\psi}$ be an entwining structure over $K$ and assume there is an entwined module $\Sigma$ such that $\Sigma_{A}$ is finitely generated and projective. We have our canonical map can : $\Sigma^{*} \otimes_{T} \Sigma \rightarrow A \otimes C$, where $T$ is the ring of endomorphisms of $\Sigma$ as an entwined module. If can is bijective we have a special type of Galois $A$-corings without grouplike elements. This process can be reversed: start with a coalgebra $C$ and a finitely generated and projective right module $\Sigma$ over an algebra $A$. Let $\rho: \Sigma \rightarrow \Sigma \otimes C$ a structure of right $C$-comodule over $\Sigma$ and define $T=\left\{t \in \operatorname{End}\left(\Sigma_{A}\right) \mid \rho(t u)=t \rho(u)\right.$ for every $\left.u \in \Sigma\right\}$ (that is, $T$ is the ring of all endomorphisms of $\Sigma$ which are $A$-linear and $C$-colinear). Then define $\operatorname{can}\left(\varphi \otimes_{T} u\right)=\sum \varphi\left(u_{(0)}\right) \otimes u_{(1)}$. If this can is bijective (which could be the new more general definition of $C$-Galois extension $T \subseteq \operatorname{End}\left(\Sigma_{A}\right)$ ) then there is a unique entwining structure $(A, C)_{\psi}$ making $\Sigma$ a right entwined module (to check this, first use propositions 2.1 and 2.5 to transfer the structure of $A$-coring of $\Sigma^{*} \otimes_{T} \Sigma$ and of right $\Sigma^{*} \otimes_{T} \Sigma$-comodule of $\Sigma$, respectively; and then [4, Proposition 2.2] to interpret everything in terms of entwining structure). Taking $\Sigma=A$, we obtain [6, Theorem 2.7].

The relationship between Noncommutative Descent Theory and Galois corings with grouplike is known (see [4] and [7]). We will derive from our analysis of Galois corings without grouplike elements a Descent Theory for ring extensions of the form $B \rightarrow \operatorname{End}\left(\Sigma_{A}\right)$, where $\Sigma_{A}$ is finitely generated and projective. Of course, our sufficient conditions to have the Descent Theorem are given on the bimodule $\Sigma$. Once again, the case $\Sigma=A$ collapses with the classical theory.

3.9. Lemma. Let ${ }_{B} \Sigma_{A}$ be a $B-A$-bimodule such that $\Sigma_{A}$ is finitely generated and projective, and let $T=\operatorname{End}\left(\Sigma_{\Sigma^{*} \otimes_{B} \Sigma}\right)$. Then the canonical map can : $\Sigma^{*} \otimes_{T} \Sigma \rightarrow \Sigma^{*} \otimes_{B} \Sigma$ is an isomorphism of $A$-corings.

Proof. Let $B \rightarrow T$ the homomorphism of rings given in Proposition 2.5. Denote by $\omega$ : $\Sigma^{*} \otimes_{B} \Sigma \rightarrow \Sigma^{*} \otimes_{T} \Sigma$ the obvious map which sends $\varphi \otimes_{B} x \mapsto \varphi \otimes_{T} x$. Let us check that $\omega$ is the inverse of can. Given $\varphi \in \Sigma^{*}$ and $x \in \Sigma$, we have $\operatorname{can}\left(\omega\left(\varphi \otimes_{B} x\right)\right)=\sum_{i} \varphi\left(e_{i}\right) e_{i}^{*} \otimes_{B} x=$ $\varphi \otimes_{B} x$, and $\omega\left(\operatorname{can}\left(\varphi \otimes_{T} x\right)=\omega\left(\sum_{i} \varphi\left(e_{i}\right) e_{i}^{*} \otimes_{B} x\right)=\omega\left(\varphi \otimes_{B} x\right)=\varphi \otimes_{T} x\right.$. Hence, can is an isomorphism of $A$-corings.

3.10. Theorem (Generalized Descent for Modules). Let ${ }_{B} \Sigma_{A}$ be a $B-A$-bimodule such that $\Sigma_{A}$ is finitely generated and projective. Then ${ }_{B} \Sigma$ is faithfully flat if and only if $-\otimes_{B} \Sigma: \mathcal{M}_{B} \rightarrow \mathcal{M}^{\Sigma^{*} \otimes_{B} \Sigma}$ is an equivalence of categories and ${ }_{A}\left(\Sigma^{*} \otimes_{B} \Sigma\right)$ is flat. In such a case, the canonical map $\lambda: B \rightarrow \operatorname{End}\left(\Sigma_{\Sigma^{*} \otimes_{B} \Sigma}\right)$ is a ring isomorphism. 
Proof. By Proposition 2.5, we have that

$$
T=\operatorname{End}\left(\Sigma_{\Sigma^{*} \otimes_{B} \Sigma}\right)=\left\{f \in \operatorname{End}\left(\Sigma_{A}\right) \mid f \otimes_{B} x=1 \otimes_{B} f(x) \text {, for every } x \in \Sigma\right\},
$$

which shows that the canonical map $B \otimes_{B} \Sigma \rightarrow T \otimes_{B} \Sigma$ is an isomorphism. Therefore, when ${ }_{B} \Sigma$ is assumed to be flat, one deduces that $\operatorname{Ker} \lambda \otimes_{B} \Sigma=\operatorname{coKer} \lambda \otimes_{B} \Sigma=0$. Thus, if ${ }_{B} \Sigma$ is faithfully flat, then $\lambda$ is an isomorphism of rings, and we can apply Lemma 3.9 and Theorem 3.2 to obtain that $-\otimes_{B} \Sigma: \mathcal{M}_{B} \rightarrow \mathcal{M}^{\Sigma^{*} \otimes_{B} \Sigma}$ is an equivalence of categories. Conversely, if we assume such an equivalence, then $\Sigma$ is a finitely generated projective generator for $\mathcal{M}^{\Sigma^{*} \otimes_{B} \Sigma}$. We deduce from Theorem 3.2 that $-\otimes_{T}: \mathcal{M}_{T} \rightarrow \mathcal{M}^{\Sigma^{*} \otimes_{B} \Sigma}$ is an equivalence of categories and $T_{T} \Sigma$ is faithfully flat. Therefore, in the commutative diagram of functors

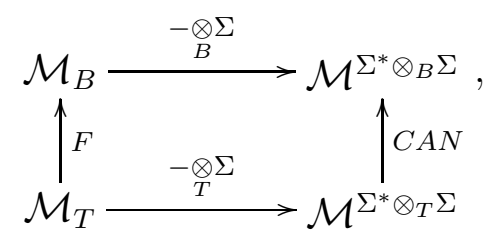

where $F: \mathcal{M}_{T} \rightarrow \mathcal{M}_{B}$ is the restriction of scalars functor associated to $\lambda: B \rightarrow T$, the other three functors are equivalences of categories. This shows that $\lambda$ is an isomorphism, which proves the Theorem.

M. Cipolla [8] give a Descent Theorem for a homomorphism of noncommutative rings $B \rightarrow A$. As T. Brzezinski pointed out [4], the category of descent data [16] is precisely the category $\mathcal{M}^{A \otimes_{B} A}$ of right comodules over $A \otimes_{B} A$. As a consequence of Theorem 3.10 we obtain Cipolla's main result [8, Teorema] (see also [16, Theorem 3.8]).

3.11. Corollary (Descent of Modules). Let $B \rightarrow A$ be a ring homomomorphism. If ${ }_{B} A$ is faithfully flat, then the functor $-\otimes_{B} A: \rightarrow \mathcal{M}^{A \otimes_{B} A}$ establishes an equivalence of categories. The converse holds if ${ }_{A}\left(A \otimes_{B} A\right)$ is flat.

Proof. Put $\Sigma=A$ in Theorem 3.10 .

\section{The structure of cosemisimple corings}

Basic properties of cosemisimple corings have been studied in 10 and 12]. Perhaps, from the coring point of view, the most fundamental examples of cosemisimple corings are Sweedler's canonical corings $D \otimes_{E} D$ for $E \subseteq D$ an extension of division rings. This section contains a full description, in terms of finitely generated and projective right $A$-modules and division subrings of their endomorphism rings, of all cosemisimple $A$-corings for each fixed ring $A$.

A coring is said to be cosemisimple if it satisfies the equivalent conditions in the following theorem. 
4.1. Theorem. [10, Theorem 3.1] Let $\mathfrak{C}$ be an A-coring. The following statements are equivalent:

(i) every left $\mathfrak{C}$-comodule is semisimple and $\mathfrak{C} \mathcal{M}$ is abelian;

(ii) every right $\mathfrak{C}$-comodule is semisimple and $\mathcal{M}^{\mathfrak{C}}$ is is abelian;

(iii) $\mathfrak{C}$ is semisimple as a left $\mathfrak{C}$-comodule and $\mathfrak{C}_{A}$ is flat;

(iv) $\mathfrak{C}$ is semisimple as a right $\mathfrak{C}$-comodule and ${ }_{A} \mathfrak{C}$ is flat;

(v) $\mathfrak{C}$ is semisimple as a right $\mathfrak{C}^{*}$-module and $\mathfrak{C}_{A}$ is projective;

(vi) $\mathfrak{C}$ is semisimple as a left ${ }^{*} \mathfrak{C}$-module and ${ }_{A} \mathfrak{C}$ is projective.

This notion obviously generalizes cosemisimple coalgebras. On the other hand, a ring $A$ is semisimple if and only if, considered as $A$-coring, $A$ is cosemisimple. In fact, cosemisimple corings were originally called semisimple corings in [10], but it seems better, from the point of view of the theory of Hopf algebras, to follow the coalgebraic terminology.

Every cosemisimple $A$-coring $\mathfrak{C}$ admits a unique decomposition as a direct sum of simple cosemisimple $A$-subcorings, where a coring is said to be simple if it has not non trivial subbicomodules [10, Theorem 3.9]. Several characterizations of simple cosemisimple corings were given in [10, Theorem 3.7]. The structure of these simple cosemisimple summands can be now deduced from our previous results.

4.2. Proposition. Let $\mathfrak{C}$ be a simple cosemisimple A-coring and $\Sigma_{\mathfrak{C}}$ a finitely generated nonzero right $\mathfrak{C}$-comodule. Let $T=\operatorname{End}\left(\Sigma_{\mathfrak{C}}\right)$ be the simple artinian ring of endomorphisms of $\Sigma$. Then $\Sigma_{A}$ is finitely generated and projective and can $: \Sigma^{*} \otimes_{T} \Sigma \rightarrow \mathfrak{C}$ is an isomorphism of A-corings. Conversely, every comatrix A-coring $\Sigma^{*} \otimes_{B} \Sigma$, where $\Sigma_{A}$ is finitely generated and projective and $B$ is simple artinian, becomes a simple cosemisimple $A$-coring, and the number of simples in a complete decomposition of $\Sigma_{\Sigma^{*} \otimes_{B} \Sigma}$ coincides with the number of simples in a complete decomposition of $B_{B}$.

Proof. The first statement is a consequence of Theorem 3.2 because $\Sigma_{\mathfrak{C}}$ is a finitely generated projective generator of $\mathcal{M}^{\mathfrak{C}}$. The second statement is a consequence of Theorem 3.10 .

The following is our structure theorem for simple cosemisimple corings.

4.3. Theorem. An A-coring $\mathfrak{C}$ is a simple cosemisimple coring if and only if there is a finitely generated and projective right $A$-module $\Sigma$ and a division subring $D \subseteq \operatorname{End}\left(\Sigma_{A}\right)$ such that $\mathfrak{C} \cong \Sigma^{*} \otimes_{D} \Sigma$ as $A$-corings. Moreover, if $\Xi$ is another finitely generated and projective right $A$-module and $E \subseteq \operatorname{End}\left(\Xi_{A}\right)$ is a division subring, then $\mathfrak{C} \cong \Xi^{*} \otimes_{E} \Xi$ as $A$-corings if and only if there is an isomorphism of right $A$-modules $g: \Sigma \rightarrow \Xi$ such that $g D g^{-1}=E$. 
Proof. That the simple cosemisimple $A$-corings are, up to isomorphism, the comatrix corings $\Sigma^{*} \otimes_{D} \Sigma$ for $\Sigma_{A}$ finitely generated and projective and $D \subseteq \operatorname{End}\left(\Sigma_{A}\right)$ a division subring, is a consequence of Proposition 4.2. Now, assume an isomorphism of $A$-corings $f: \Sigma^{*} \otimes_{D} \Sigma \rightarrow \Xi^{*} \otimes_{E} \Xi$ as stated, and let $(-)_{f}: \mathcal{M}^{\Sigma^{*} \otimes_{D} \Sigma} \rightarrow \mathcal{M}^{T^{*} \otimes_{E} T}$ the associated induction functor [11, 5.2], which is an isomorphism of categories. By Proposition 4.2, $\Sigma$ is a simple right $\Sigma^{*} \otimes_{D} \Sigma$-comodule, which implies that $\Sigma_{f}$ is a simple right $\Xi^{*} \otimes_{E} \Xi-$ comodule. Since $\Xi$ is, up to isomorphism, the only simple comodule over $\Xi^{*} \otimes_{E} \Xi$, there is an isomorphism of comodules $g: \Sigma_{f} \rightarrow \Xi$, which, at the level of right $A$-modules, gives an isomorphism $g: \Sigma_{A} \rightarrow \Xi_{A}$. Clearly, $\operatorname{End}\left(\left(\Sigma_{f}\right)_{\Xi^{*} \otimes_{E} \Xi}\right)=\operatorname{End}\left(\Sigma_{\Sigma^{*} \otimes_{D} \Sigma}\right)$. By Theorem 3.10, $D=\operatorname{End}\left(\Sigma_{\Sigma^{*} \otimes_{D} \Sigma}\right)$ and $E=\operatorname{End}\left(\Xi_{\Xi^{*} \otimes_{E} \Xi}\right)$. Thus, if $d \in D$, then $g d g^{-1}$ is an endomorphism of the comodule $\Xi$, that is, $g d g^{-1} \in E$. We have obtained that $g D g^{-1} \subseteq E$. The other inclusion is also easily obtained. Conversely, assume that $g: \Sigma \rightarrow \Xi$ is an isomorphism of right $A$-modules such that $g D g^{-1}=E$, and consider the following $A$-bilinear map

$$
\psi: \Sigma^{*} \times \Sigma \rightarrow \Xi^{*} \otimes_{E} \Xi, \quad\left((\varphi, u) \mapsto \varphi g^{-1} \otimes_{E} g(u)\right) .
$$

Let $d \in D$ and $e=g d g^{-1} \in E$, so $\psi(\varphi d, u)=\left(\varphi d g^{-1}\right) \otimes_{E} g(u)=\varphi g^{-1} e \otimes_{E} g(u)=$ $\varphi g^{-1} \otimes_{E} e g(u)=\varphi g^{-1} \otimes_{E} g(d u)=\psi(\varphi, d u)$. Hence $\psi$ extended to an $A$-bilinear map $\psi: \Sigma^{*} \otimes_{D} \Sigma \rightarrow \Xi^{*} \otimes_{E} \Xi$. Given any right dual basis $\left\{e_{i}^{*}, e_{i}\right\}$ for $\Sigma_{A}$, it is easy to see that $\left\{e_{i}^{*} \circ g^{-1}, g\left(e_{i}\right)\right\}$ is a right dual basis for $\Xi_{A}$. Moreover, $\psi\left(e_{i}^{*} \otimes_{D} e_{j}\right)=\left(e_{i}^{*} \circ g^{-1}\right) \otimes_{E} g\left(e_{j}\right)$, for all $i, j$, thus $\psi$ is an isomorphism of $A$-bimodules. A direct computation, using these bases, proves that $\psi$ is a morphism of $A$-corings.

Recall from [10, Theorem 3.7] that an $A$-coring $\mathfrak{C}$ is cosemisimple if and only if it decomposes uniquely as $\mathfrak{C}=\oplus_{\lambda \in \Lambda} \mathfrak{C}_{\lambda}$, where $\mathfrak{C}_{\lambda}$ are simple cosemisimple $A$-corings.

4.4. Theorem (Structure of cosemisimple corings). Let $\mathfrak{C}$ be an $A$-coring. The following statements are equivalents

(i) $\mathfrak{C}$ is a cosemisimple A-coring;

(ii) there exists a family $\Lambda$ of finitely generated projective right $A$-modules, and a division subring $D_{\Sigma} \subseteq \operatorname{End}\left(\Sigma_{A}\right)$, for every $\Sigma \in \Lambda$, such that $\mathfrak{C} \cong \oplus_{\Sigma \in \Lambda}\left(\Sigma^{*} \otimes_{D_{\Sigma}} \Sigma\right)$ as $A$ corings.

Furthermore, if $\mathfrak{C}$ satisfies one of these conditions, then the family $\Lambda$ is a set of representatives of all simple right $\mathfrak{C}$-comodules, and the decomposition is unique in the following sense: given any other family $\Lambda^{\prime}$ satisfying $($ ii $)$; then there exists a bijective map $\phi: \Lambda \rightarrow \Lambda^{\prime}$ and an isomorphism of right $A$-modules $g_{\Sigma}: \Sigma_{A} \rightarrow \phi\left(\Sigma_{A}\right)$ for every $\Sigma \in \Lambda$ such that $D_{\phi(\Sigma)}=g_{\Sigma}\left(D_{\Sigma}\right) g_{\Sigma}^{-1}$.

Proof. The equivalence $(i) \Leftrightarrow(i i)$ follows from [10, Theorem 3.7] and Theorem 4.3. Let us check the uniqueness of the decomposition. Let $\Lambda, \Lambda^{\prime}$ to be as in $(i i)$ with the associated isomorphisms of $A$-corings

$$
\chi: \mathfrak{C} \rightarrow \oplus_{\Sigma \in \Lambda}\left(\Sigma^{*} \otimes_{D_{\Sigma}} \Sigma\right), \quad \zeta: \mathfrak{C} \rightarrow \oplus_{\Sigma^{\prime} \in \Lambda^{\prime}}\left(\Sigma^{*} \otimes_{D_{\Sigma^{\prime}}} \Sigma^{\prime}\right) .
$$


Therefore, $\mathfrak{C}=\oplus_{\Sigma \in \Lambda} \mathfrak{C}_{\Sigma}=\oplus_{\Sigma^{\prime} \in \Lambda^{\prime}} \mathfrak{C}_{\Sigma^{\prime}}$, where $\mathfrak{C}_{\Sigma}=\chi^{-1}\left(\Sigma^{*} \otimes_{D_{\Sigma}} \Sigma\right), \mathfrak{C}_{\Sigma^{\prime}}=\zeta^{-1}\left(\Sigma^{*} \otimes_{D_{\Sigma^{\prime}}} \Sigma^{\prime}\right)$ are simple cosemisimple $A$-corings for every $\Sigma \in \Lambda$ and $\Sigma^{\prime} \in \Lambda^{\prime}$. The uniqueness of the decomposition given in [10, Theorem 3.7] gives now that there exists a bijection $\phi: \Lambda \rightarrow \Lambda^{\prime}$ such that $\mathfrak{C}_{\Sigma}=\mathfrak{C}_{\phi(\Sigma)}$ for every $\Sigma \in \Lambda$. That is, $\Sigma^{*} \otimes_{D_{\Sigma}} \Sigma \cong \phi(\Sigma)^{*} \otimes_{D_{\phi(\Sigma)}} \phi(\Sigma)$, as $A^{-}$ corings, for every $\Sigma \in \Lambda$. This implies in view of Theorem 4.3 that there exists an $A$-linear isomorphism $g_{\Sigma}: \Sigma \rightarrow \phi(\Sigma)$ such that $D_{\phi(\Sigma)}=g_{\Sigma}\left(D_{\Sigma}\right) g_{\Sigma}^{-1}$.

4.5. Remark. The structure of cosemisimple coalgebras over a field $k$ is very well-known: form direct sums of dual coalgebras of finite dimensional simple algebras over $k$, which are matrices over division $k$-algebras. Comatrix corings allow to built these simple blocks directly, as they are coalgebras of the form $\Sigma^{*} \otimes_{D_{\Sigma}} \Sigma$, where the $D_{\Sigma}$ 's are division subalgebras of endomorphism algebras of finite dimensional $k$-vector spaces $\Sigma$. Observe that this description applies for coalgebras over arbitrary commutative rings $k$, if we take finitely generated and projective $k$-modules instead of finite dimensional vector spaces.

\section{Coendomorphism corings}

We will see that comatrix corings are special instances of coendomorphism corings. This gives an alternative approach, although less elementary, for introducing comatrix corings and the canonical map.

Let $\mathfrak{C}$ and $\mathfrak{D}$ be an $A$-coring and $B$-coring, respectively. Let $N$ be an $A-B$-bimodule with a right $\mathfrak{D}$-coaction map $\rho_{N}: N \rightarrow N \otimes_{B} \mathfrak{D}$ which is left $A$-linear. Assume that $N_{\mathfrak{D}}$ is quasi-finite, that is, the functor $-\otimes_{A} N: \mathcal{M}_{A} \rightarrow \mathcal{M}^{\mathfrak{D}}$ has a left adjoint $F$ : $\mathcal{M}^{\mathfrak{D}} \rightarrow \mathcal{M}_{A}$ (see [11, Section 3]). This functor is called the cohom functor by analogy with the case of coalgebras over fields (see [19]); notation $F=\mathrm{h}_{\mathfrak{D}}(N,-)$. Let $\eta_{-,-}$: $\operatorname{Hom}_{\mathfrak{D}}\left(-,-\otimes_{A} N\right) \rightarrow \operatorname{Hom}_{A}(F(-),-)$ denote the natural isomorphism of the adjunction, and $\theta: 1_{\mathcal{M}^{\mathcal{D}}} \rightarrow F(-) \otimes_{A} N$ the unity of the adjunction. The canonical map $A_{A} \rightarrow$ $\operatorname{Hom}_{\mathfrak{D}}(N, N) \rightarrow \operatorname{Hom}_{A}(F(N), F(N))$ gives a structure of left $A$-module on $F(N)$ such that $F(N)$ becomes an $A$-bimodule. Define a comultiplication $\Delta: F(N) \rightarrow F(N) \otimes_{A} F(N)$ by $\Delta=\eta_{N, F(N) \otimes_{A} F(N)}\left(\left(F(N) \otimes_{A} \theta_{N}\right) \circ \theta_{N}\right)$, that is, $\Delta$ is determined by the condition $\left(F(N) \otimes_{A} \theta_{N}\right) \circ \theta_{N}=\left(\Delta \otimes_{A} N\right) \circ \theta_{N}$; clearly $\Delta$ is $A$-bilinear. An analogous proof to that of [2, Proposition III.3.1] shows that $\Delta$ is coassociative. Moreover, $F(M)$ becomes an $A$-coring with the counit given by $\epsilon=\eta_{N, A}(\iota)$, where $\iota: N \rightarrow A \otimes_{A} N$ is the canonical isomorphism. This $A$-coring will be denoted by $e_{\mathfrak{D}}(N)$; we refer to it as the coendomorphism $A$-coring associated to $N_{\mathfrak{D}}$.

5.1. Example. Let $\mathfrak{C}$ be an $A$-coring, and $\left(\Sigma, \rho_{\Sigma}\right)$ a right $\mathfrak{C}$-comodule such that $\Sigma_{A}$ is finitely generated and projective with finite right dual basis $\left\{e_{i}^{*}, e_{i}\right\}$; denote by $T=$ $\operatorname{End}\left(\Sigma_{\mathfrak{C}}\right)$ the endomorphism ring of $\Sigma_{\mathfrak{C}}$. It is easy to check that the $A$-linear map

$$
\lambda_{\Sigma^{*}}: \Sigma^{*} \rightarrow \mathfrak{C} \otimes_{A} \Sigma^{*}, \quad\left(\varphi \mapsto \sum_{i}\left(\left(\varphi \otimes_{A} \mathfrak{C}\right) \rho_{\Sigma}\right)\left(e_{i}\right) \otimes_{A} e_{i}^{*}\right)
$$


endows $\Sigma^{*}$ with a structure of left $\mathfrak{C}$-comodule. Moreover, $(-)^{*}: T \rightarrow \operatorname{End}\left(\mathfrak{C}^{\Sigma^{*}}\right)$ sending $f \mapsto f^{*}$ establishes a ring isomorphism. Hence $\Sigma^{*}$ is a $\mathfrak{C}-T$-bicomodule. There is a canonical adjunction $-\otimes_{T} \Sigma \dashv-\otimes_{A} \Sigma^{*}$; the associated natural isomorphism is

$$
\begin{gathered}
\eta_{Y_{T}, X_{A}}: \operatorname{Hom}_{T}\left(Y, X \otimes_{A} \Sigma^{*}\right) \longrightarrow \operatorname{Hom}_{A}\left(Y \otimes_{T} \Sigma, X\right) \\
\quad f(y)=\sum_{i} x_{i} \otimes_{A} e_{i}^{*} \longmapsto\left(y \otimes_{T} u \mapsto \sum_{i} x_{i} e_{i}^{*}(u)\right) \\
\left(y \mapsto \sum_{i} g\left(y \otimes_{T} e_{i}\right) \otimes_{A} e_{i}^{*}\right) \longleftrightarrow g
\end{gathered}
$$

the unit and counit are given by

$$
\begin{aligned}
\theta_{Y_{T}}: Y \longrightarrow Y \otimes_{T} \Sigma \otimes_{A} \Sigma^{*} & \chi_{X_{A}}: X \otimes_{A} \Sigma^{*} \otimes_{T} \Sigma \longrightarrow X \\
y & \longmapsto \sum_{i} y \otimes_{B} e_{i} \otimes_{A} e_{i}^{*}, \quad x \otimes_{A} e_{i}^{*} \otimes_{T} e_{j} \longmapsto x e_{i}^{*}\left(e_{j}\right) .
\end{aligned}
$$

Therefore, $\Sigma_{T}^{*}$ is quasi-finite (see [11, Example 3.4]). In this way, the coendomorphism $A$-coring associated to $\Sigma_{T}^{*}$ is then $e_{T}\left(\Sigma^{*}\right)=\Sigma^{*} \otimes_{T} \Sigma$ with the following comultiplication and counit

$$
\begin{aligned}
\Delta: e_{T}\left(\Sigma^{*}\right) \rightarrow e_{T}\left(\Sigma^{*}\right) \otimes_{A} e_{T}\left(\Sigma^{*}\right), \quad\left(\varphi \otimes_{T} u \mapsto \sum_{i, j} \varphi \otimes_{T} e_{i} \otimes_{A} e_{i}^{*} \otimes_{T} e_{j} e_{j}^{*}(u)\right. & \\
& \left.=\sum_{i} \varphi \otimes_{T} e_{i} \otimes_{A} e_{i}^{*} \otimes_{T} u\right),
\end{aligned}
$$

$\epsilon: \Sigma^{*} \otimes_{T} \Sigma \rightarrow A$ sending $\varphi \otimes_{T} u \mapsto \sum_{i} \varphi\left(e_{i}\right) e_{i}^{*}(u)=\varphi(u)$. We have shown that $e_{T}\left(\Sigma^{*}\right)$ is just the comatrix $A$-coring of ${ }_{T} \Sigma_{A}$.

Now, assume that $N$ is a $\mathfrak{C}-\mathfrak{D}$-bicomodule and that ${ }_{B} \mathfrak{D}$ is a flat module. By $[11$, Proposition 3.3], $F=\mathrm{h}_{\mathfrak{D}}(N,-)$ factorizes throughout the category $\mathcal{M}^{\mathfrak{C}}$, and $\mathrm{h}_{\mathfrak{D}}(N,-)$ : $\mathcal{M}^{\mathfrak{D}} \rightarrow \mathcal{M}^{\mathfrak{C}}$ becomes a left adjoint to the cotensor product functor $-\square_{\mathfrak{C}} N: \mathcal{M}^{\mathfrak{C}} \rightarrow \mathcal{M}^{\mathfrak{D}}$ with unity $\theta: 1_{\mathcal{M}^{\mathfrak{P}}} \rightarrow F(-) \square_{\mathfrak{C}} N$ and counity $\chi: F\left(-\square_{\mathfrak{C}} N\right) \rightarrow 1_{\mathcal{M}^{\mathfrak{C}}}$.

5.2. Proposition. Let $N$ be a $\mathfrak{C}-\mathfrak{D}$-bicomodule that is quasi-finite as a right $\mathfrak{D}$-comodule, and assume that ${ }_{B} \mathfrak{D}$ is flat. The map $f: e_{\mathfrak{D}}(N) \rightarrow \mathfrak{C}$ defined by $f=\chi_{\mathfrak{C}} \circ \mathrm{h}_{\mathfrak{D}}\left(N, \lambda_{N}\right)$, where $\lambda_{N}: N \rightarrow \mathfrak{C} \otimes_{A} N$ is the left comodule structure map, is a homomorphism of $A$-corings.

Proof. The following diagram

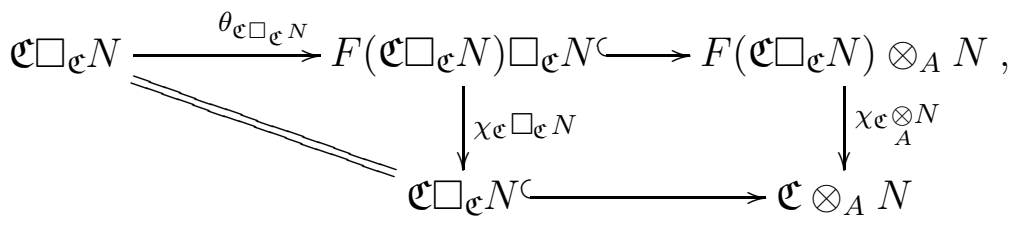

where the hooked arrows are canonical monomorphisms, is commutative, because the left triangle commutes by the properties of the unity and counity of the adjunction. We have 
then that the diagram

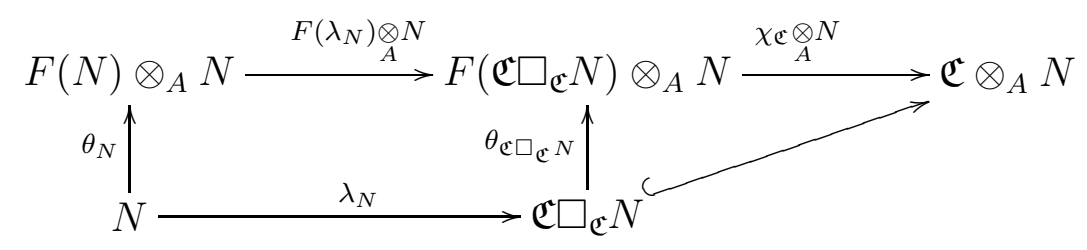

is commutative. This means that $\left(f \otimes_{A} N\right) \circ \theta_{N}=\lambda_{N}$. To prove that $f$ is a homomorphism of $A$-corings, we need to show that the following diagram is commutative:

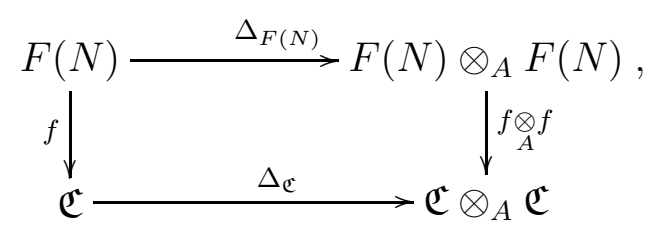

which is equivalent, by the adjunction isomorphism, to prove that the diagram

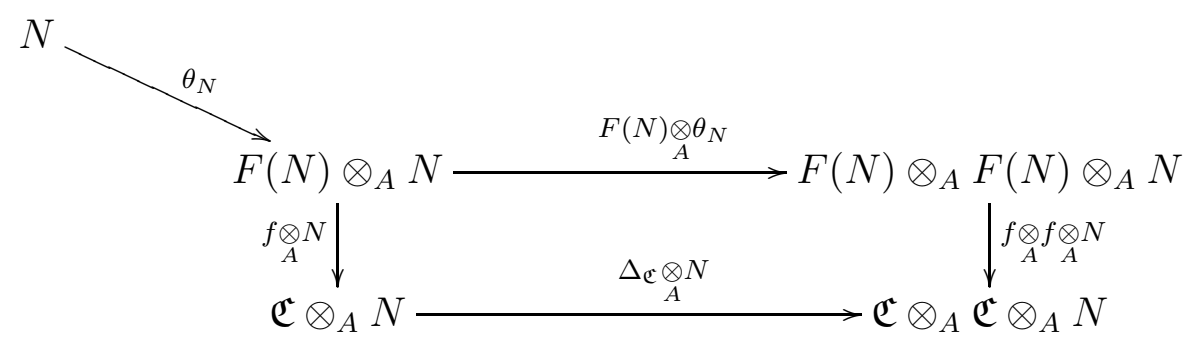

is commutative. So

$$
\begin{gathered}
\left(\Delta_{\mathfrak{C}} \otimes_{A} N\right) \circ\left(f \otimes_{A} N\right) \circ \theta_{N}=\left(\Delta_{\mathfrak{C}} \otimes_{A} N\right) \circ \lambda_{N}=\left(\mathfrak{C} \otimes_{A} \lambda_{N}\right) \circ \lambda_{N} ; \\
\left(f \otimes_{A} f \otimes_{A} N\right) \circ\left(F(N) \otimes_{A} \theta_{N}\right) \circ \theta_{N}=\left(f \otimes_{A}\left(\left(f \otimes_{A} N\right) \circ \theta_{N}\right)\right) \circ \theta_{N} \\
=\left(f \otimes_{A} \lambda_{N}\right) \circ \theta_{N}=\left(\mathfrak{C} \otimes_{A} \lambda_{N}\right) \circ\left(f \otimes_{A} N\right) \circ \theta_{N}=\left(\mathfrak{C} \otimes_{A} \lambda_{N}\right) \circ \lambda_{N}
\end{gathered}
$$

Therefore, (11) is commutative, and so is (10). Finally, we have to show that $\epsilon_{\mathfrak{C}} \circ f=\epsilon_{F(N)}$, which is equivalent to show that $\left(\epsilon_{\mathfrak{C}} \otimes_{A} N\right) \circ\left(f \otimes_{A} N\right) \circ \theta_{N}=\left(\epsilon_{F(N)} \otimes_{A} N\right) \circ \theta_{N}$. This is clear from the commutative diagram

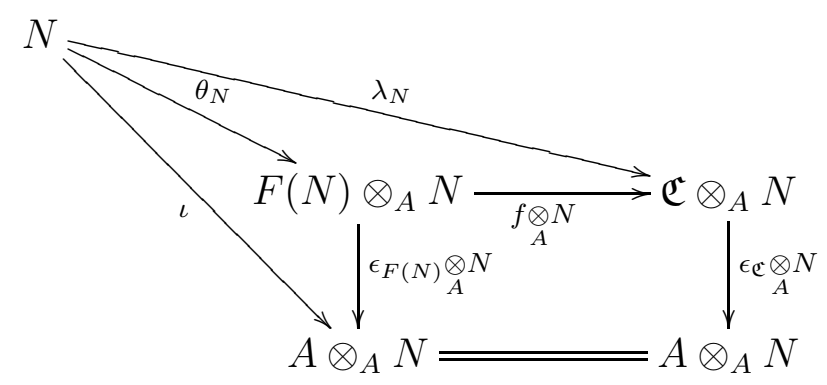


5.3. Example. Let $\mathfrak{C}, \Sigma$ and $T$ as in Example 5.1. Then, by Proposition 5.2, there exists an $A$-coring morphism $f=\chi_{\mathfrak{C}} \circ\left(\lambda_{\Sigma^{*}} \otimes_{T} \Sigma\right): e_{T}\left(\Sigma^{*}\right) \rightarrow \mathfrak{C}$. Using the notation of Example 5.1, we have $\left.f\left(\varphi \otimes_{T} u\right)=\sum_{i} \chi_{\mathfrak{c}}\left(\left(\varphi \otimes_{A} \mathfrak{C}\right) \circ \rho_{\Sigma}\left(e_{i}\right)\right) \otimes_{A} e_{i}^{*} \otimes_{T} u\right)=\sum_{i}\left(\varphi \otimes_{A} \mathfrak{C}\right) \rho_{\Sigma}\left(e_{i}\right) e_{i}^{*}(u)=$ $\left(\varphi \otimes_{A} \mathfrak{C}\right) \circ \rho_{\Sigma}(u)$. Therefore, $f=$ can, the morphism of $A$-corings defined in Proposition 2.7 .

\section{References}

[1] J. Y. Abuhlail, Morita contexts for corings and equivalences, preprint, 2002.

[2] K. Al Takhman, Äquivalenzen zwischen Komodulkategorien von Koalgebran über Ringen, Ph.D. thesis, Univ. Düsseldorf, 1999.

[3] T. Brzeziński, On modules associated to coalgebra Galois extensions, J. Algebra 215 (1999), 290-317.

[4] T. Brzeziński, The structure of corings. Induction functors, Maschke-type theorem, and Frobenius and Galois-type properties, preprint math.RA/0002105, to appear in Algebras and Representation Theory.

[5] T. Brzeziński, The structure of corings with a grouplike element, preprint arXiv:math.RA/0108117, 2001.

[6] T. Brzeziński and P. M Hajac, Coalgebras extensions and algebras coextensions of galois type, Commun. Algebra 27 (1999), 1347-1367.

[7] S. Caeneepel, J. Vercruysse, and S. Wang, Morita Theory for corings and cleft entwining structures, preprint arXiv:math.RA/0206198v1, 2002.

[8] M. Cipolla, Discesa fedelmente piatta dei moduli, Ren. Cric. Mat. Palermo 25 (1976), no. $2,43-46$.

[9] F.R. DeMeyer, Some notes on the general Galois theory of rings, Osaka J. Math. 2 (1965), 117-127.

[10] L. EL Kaoutit, J. Gómez-Torrecillas, and F. J. Lobillo, Semisimple corings, preprint arXiv:math.RA/0201070v1, to appear in Algebra Colloquium.

[11] J. Gómez-Torrecillas, Separable functors in corings, IJMMS 30 (2002), 203-225.

[12] J. Gómez-Torrecillas and A. Louly, preprint arXiv:math.RA/0206175, 2002.

[13] F. Guzman, Cointegrations, relative cohomology for comodules, and coseparable corings, J. Algebra 126 (1989), 211-224.

[14] T. Kanzaki, On commutor rings and Galois theory of separable algebras, Osaka J. Math. 1 (1964), 103-115. 
[15] M. Kleiner, The dual ring to a coring with a grouplike, Proc. Amer. Math. Soc. 91 (1984), 540-542.

[16] P. Nuss, Noncommutative descent and non-abelian cohomology, K-Theory 12 (1997), $23-74$.

[17] H.J. Schneider, Principal homogeneous spaces for arbitrary Hopf algebras, Israel. J. Math. 72 (1990), no. 1-2, 167-195.

[18] M. Sweedler, The predual theorem to the Jacobson-Bourbaki Theorem, Trans. Amer. Math. Soc. 213 (1975), 391-406.

[19] M. Takeuchi, Morita theorems for categories of comodules, J. Fac. Sci. Univ. Tokyo 24 (1977), 629-644. 